Received: $\quad 2019.07 .05$ Accepted: 2019.08.22 Published: 2020.01 .08
Authors' Contribution: Study Design A Data Collection B Statistical Analysis C Data Interpretation D Manuscript Preparation E Literature Search F Funds Collection G

\section{N-myc Downstream-Regulated Gene 2 (NDRG2) Promotes Bone Morphogenetic Protein 2 (BMP2)-Induced Osteoblastic Differentiation and Calcification by Janus Kinase 3 (JAK3)/Signal Transducer and Activator of Transcription 3 (STAT3) Signaling Pathway}

BCDE 1 SUnYu Chen

BC 1 JianKun Wang

BC 1 Chao Cai

AF 2 Xiaoyan Xie
1 Department of Orthopedics, Fuzhou Second Hospital Affiliated to Xiamen University, Fuzhou, Fujian, P.R. China

2 Department of Internal Medicine, Clinical Medical College of Jining Medical University, Jining, Shandong, P.R. China
Corresponding Author: Source of support:
Xiaoyan Xie, e-mail: xyxie3090@163.com

Departmental sources

Background: Osteoporosis is an osteolytic disease resulted from imbalance in bone homeostasis. Studies indicated that $\mathrm{N}$-myc downstream-regulated gene 2 (NDRG2) could affect the osteoclast differentiation. However, the effect of NDRG2 on osteoblastic differentiation and calcification remains unknown. Hence, we aimed to analyze the effect of NDRG2 on the proliferation and differentiation of osteoblasts.

Material/Methods: The differentiation of bone morphogenetic protein 2 (BMP2) induced MC3T3-E1 cells was observed by the microscope. Real-time quantitative polymerase chain reaction (RT-qPCR) and western blot analysis detected the expression of BMP2, NDRG2, runt-related transcription factor 2 (RunX2), osteoprotegerin (OPG), osterix (OSX), and osteocalcin (OCN). Alkaline phosphatase (ALP) activity assay was detecting the ALP activity and alizarin red staining assay was analyzing intracellular calcium salt deposition. The cell transfection was also verified by RT-qPCR analysis.

Results: The results demonstrated that BMP2 promoted the osteoblastic differentiation with the increasing expression of Runx2, OPG, OSX, and OCN. NDRG2 expression was upregulated during osteogenic differentiation. NDRG2 overexpression promoted the expression of Runx2, OPG, OSX, and OCN, and increased the ALP activity while NDRG2 inhibition reversed the changes. NDRG2 overexpression increased the intracellular calcium salt deposition and NDRG2 inhibition reversed the changes. The role of NDRG2 in osteoblastic differentiation and calcification was played through the JAK3/STAT3 signal pathway.

Conclusions: The presented data indicated that NDRG2 promoted BMP2-induced osteoblastic differentiation and calcification by activating the JAK3/STAT3 signal pathway.

MeSH Keywords:

Bone Morphogenetic Protein Receptors, Type II • Calcification, Physiologic • Cell Differentiation • Osteoblasts

Full-text PDF: https://www.medscimonit.com/abstract/index/idArt/918541

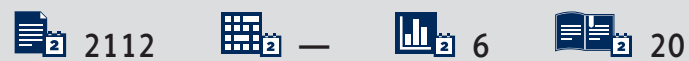




\section{Background}

Osteoporosis is a systemic metabolic osteopathy characterized by the decreased bone mass and degradation of bone microstructure. The osteoporosis risk is estimated to be approximately $72 \%$ for women and $62 \%$ for men, who are older than 50 years of age [1,2]. With the speeding up of aging, osteoporosis has become a common and frequently occurring disease in the world. According to the results of China's 2013 population census, the number of patients with osteoporosis or low bone density in China will reach 212 million [3]. The absolute number of osteoporosis patients in China shows an obvious rising trend, seriously endangering the health and quality of life of middle-aged and elderly people. Although some drugs for the treatment of osteoporosis have curative effects, some drugs still have the varying degree side effect. Therefore, it is extremely urgent to find a new drug for the effective treatment of osteoporosis.

$\mathrm{N}$-myc downstream regulator gene 2 (NDRG2) is involved in cell growth and differentiation hormone response [4-6]. Tamura et al. [7] studied role of NDRG2 in oral squamous cell carcinoma and found that NDRG2 overexpression inhibited the PI3K/AKT and NF-KB signaling pathways to suppress the epithelial-mesenchymal transformation of oral squamous cell carcinoma. NDRG2 is also related to osteoclast differentiation. Kim et al. [8] indicated that NDRG2 could inhibit the breast cancer induced osteoclast differentiation by downregulation of intercellular adhesion molecule 1 (ICAM1). Kang et al. [9] showed that NDRG2 potentially inhibited osteoclast differentiation and regulated the signal transduction pathway related to osteoclastogenesis. However, its role in osteoblast differentiation is unknown.

Hence, we aimed to analyze the effect of NDRG2 on the proliferation and differentiation of osteoblasts.

\section{Material and Methods}

\section{Cell culture and cell treatment}

MC3T3-E1 cells were purchased from American Type Culture Collection (Rockville, MD, USA) and grew in DMEM medium containing $15 \%$ fetal bovine serum in an environment containing $5 \% \mathrm{CO}_{2}$ at $37^{\circ} \mathrm{C}$. MC3T3-E1 cells were induced by $300 \mathrm{ng} / \mathrm{mL}$ bone morphogenetic protein 2 (BMP2) for 14 days and cell differentiation was observed by a microscope at day 0,7 , and 14 .

\section{Real-time quantitative polymerase chain reaction (RT-qPCR) analysis}

MC3T3-E1 cells were collected after BMP2 induction or transfection. Total RNA of cells was extracted with TRIzol kit (Invitrogen; Thermo Fisher Scientific, Inc.), and its concentration and purity were detected. The cDNA was synthesized with reverse transcription kit (Takara Biotechnology Co., Ltd., Beijing, China), and the operation procedure was strict in accordance with the instructions of reverse transcription kit. After the concentration of cDNA was adjusted, the Master Mix of SYBR Green RT-qPCR (Invitrogen; Thermo Fisher Scientific, Inc.) was used for PCR reaction. Reaction condition is as follows: $94^{\circ} \mathrm{C}$ predenaturation for 5 minutes, $94^{\circ} \mathrm{C}$ denaturation for 10 seconds, $56^{\circ} \mathrm{C}$ annealing for 30 seconds, $72^{\circ} \mathrm{C}$ extension for 30 seconds, a total of 40 cycles, and $72^{\circ} \mathrm{C}$ terminal extension for $10 \mathrm{~min}$ utes. GAPDH was an internal control and the $2^{-\Delta \Delta C t}$ method to calculate the mRNA expression of runt-related transcription factor 2 (Runx2), osteoprotegerin (OPG), osterix (OSX), and osteocalcin (OCN) and NDRG2 in MC3T3-E1 cells. The primer sequences for $\mathrm{qPCR}$ were as follows:

GAPDH forward, 5'-AAGTTCAACGGCACAGTCAAGG-3', and reverse, 5'-ACGCCAGTAGACTCCACGACAT-3'; Runx2 forward, 5'-GAACCAAGAAGGCACAGACAGAA-3', and reverse, 5'-GGCGGGACACCTACTCTCATACT-3'; OPG forward, 5'-CCTTGCCCTGACCACTAC-3', and reverse, 5'-TCATTTGAGAAGAACCCATC-3'; OSX forward, 5'-GACTCAACAGCCCTGGGAAAA-3', and reverse, 5'-GGGTGGGTAGTCATTGGCATAG-3'; OCN forward, 5'-GCCCTCACACTCCTCGCC-3', and reverse, 5'-TCTTCACTACCTCGCTGCCC-3'; NDRG2 forward, 5'-CAGGACAAACACCCGAGACT-3', and reverse, 5'-AGCCATAAGGTGTCTCCACAG-3'.

\section{Western blot analysis}

After phosphate-buffered saline (PBS) washed the cells with PBS for 3 times, the pre-cooled lysate was added to MC3T3-E1 cells, which was placed on ice for full lysis and centrifuged at $48000 \mathrm{rpm}$ for 10 minutes to remove the supernatant. The protein concentration was determined with bicinchoninic acid (BCA) protein concentration detection kit (Pierce; Thermo Fisher Scientific, Inc.). The protein was mixed with loading buffer with a volume ratio of 1: 4 , which was heated in boiling water for 10 minutes to make protein denaturation. $30 \mu \mathrm{g}$ denatured protein samples per hole was performed with gel electrophoresis separation (100 g/L separating gel and $50 \mathrm{~g} / \mathrm{L}$ stacking gel) for 2 hours at the electrophoretic conditions of $110 \mathrm{~V}$ constant voltage. After electrophoresis, western transfer was conducted at $250 \mathrm{~mA}$ constant flow at $4^{\circ} \mathrm{C}$ for 2 hours. After that, membrane was incubated in the $50 \mathrm{~g} / \mathrm{L}$ of bovine serum albumin at $25^{\circ} \mathrm{C}$ for 2 hours. Then, membrane was incubated overnight at $4^{\circ} \mathrm{C}$ with primary 


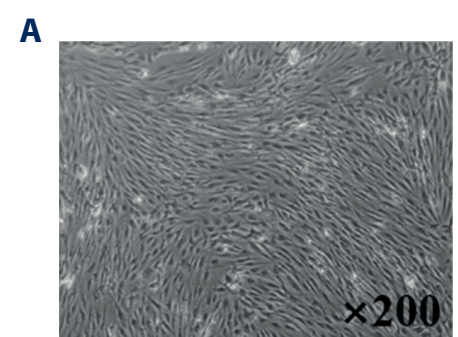

$\mathrm{Oh}$

B
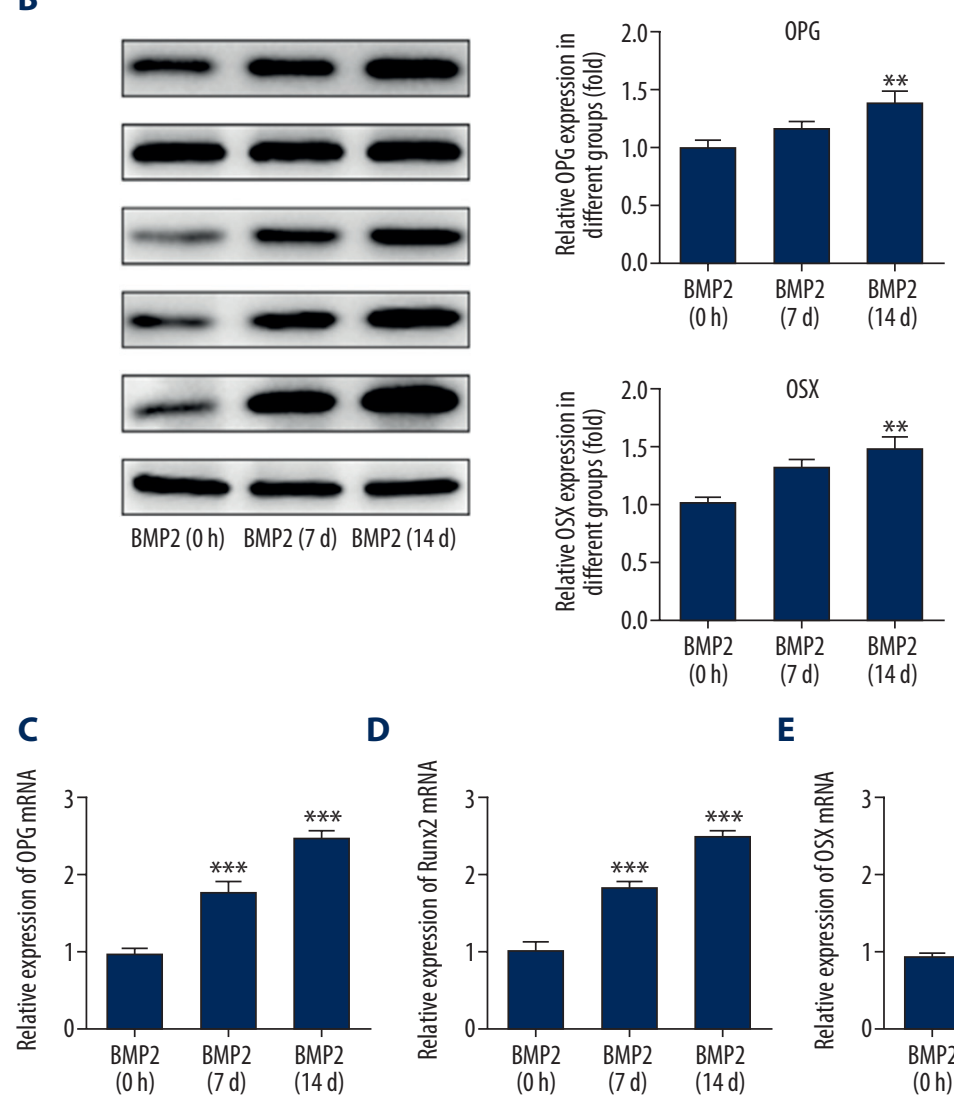

D

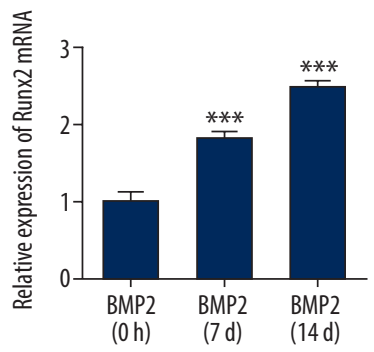

$\mathbf{E}$

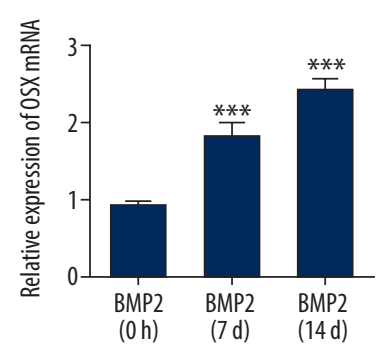

$\mathbf{F}$

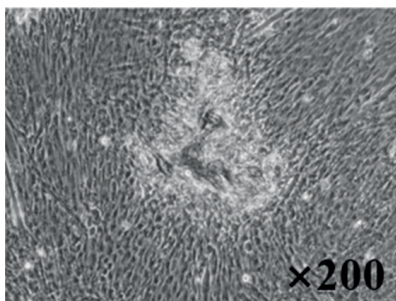

$14 \mathrm{~d}$
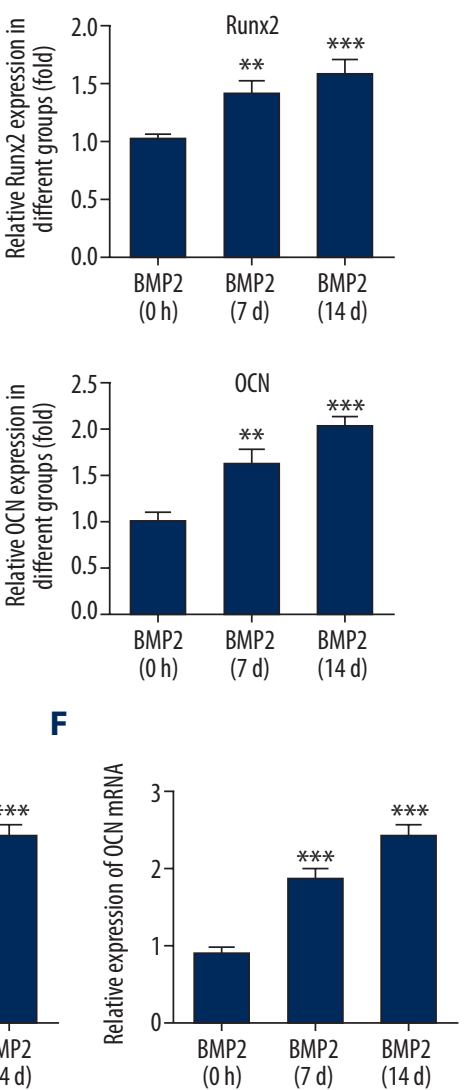

H

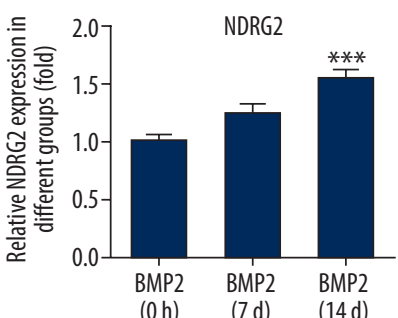

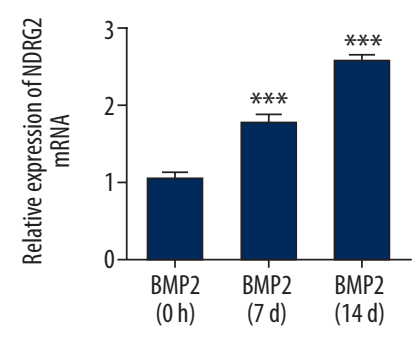

Figure 1. NDRG2 expression was increased in BMP2-induced MC3T3-E1 cells. (A) The cell differentiation was increased after BMP2 induction with time. (B) The expression of Runx2, OPG, OSX, and OCN was increased after BMP2 induction with time. ${ }^{* *} P<0.01$ and ${ }^{* *} P<0.001$ versus BMP2 ( 0 h) group. (C-F) The mRNA expression of Runx2, OPG, OSX, and OCN was increased after BMP2 induction with time. ${ }^{* * *} P<0.001$ versus BMP2 (Oh) group. (G) The expression of NDRG2 was increased after BMP2 induction with time. ${ }^{*} P<0.05$ and ${ }^{* * *} P<0.001$ versus BMP2 (Oh) group. (H) The mRNA expression of NDRG2 was increased after BMP2 induction with time. ${ }^{* * *} P<0.001$ versus BMP2 $(0 \mathrm{~h})$ group. 
A

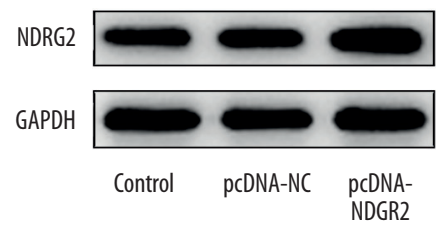

C

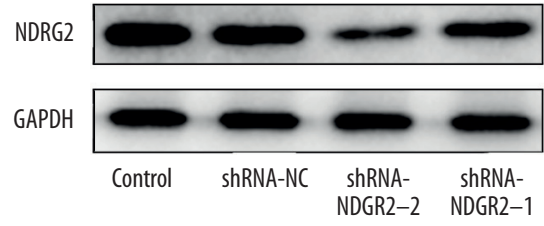

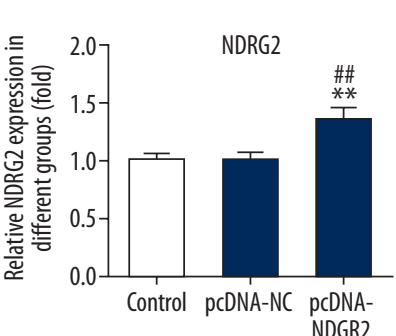

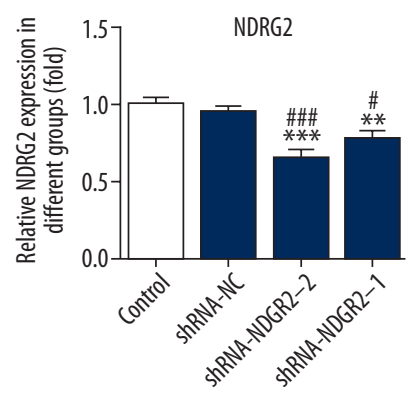

B

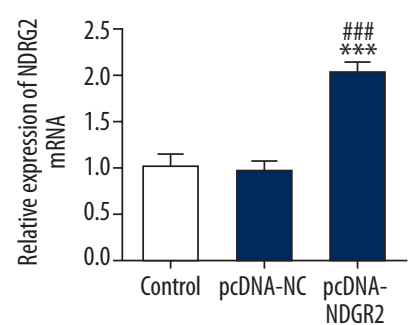

D

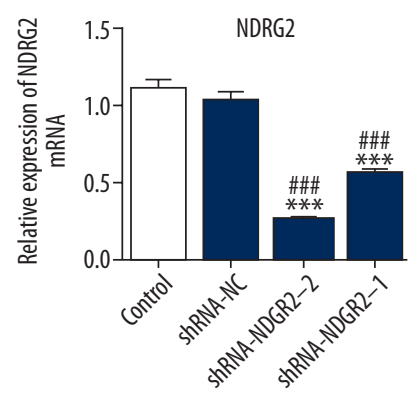

Figure 2. NDRG2 was increased or decreased in MC3T3-E1 cells after transfected with pCDNA-NDRG2 or shRNA-NDRG2.

(A) The expression of NDRG2 was increased in MC3T3-E1 cells after transfected with pCDNA-NDRG2. ${ }^{* *} P<0.01$ versus control group. \#\# $P<0.01$ versus pcDNA-NC group. (B) The mRNA expression of NDRG2 was increased in MC3T3-E1 cells after transfected with pcDNA-NDRG2. ${ }^{* * *} P<0.001$ versus control group. ${ }^{\# \# \#} P<0.001$ versus pcDNA-NC group. (C) The expression of NDRG2 was decreased in MC3T3-E1 cells after transfected with shRNA-NDRG2. ${ }^{* *} P<0.01$ and ${ }^{* * *} P<0.001$ versus control group. $\# P<0.001$ and \#\#\# $P<0.001$ versus shRNA-NC group. (D) The mRNA expression of NDRG2 was decreased in MC3T3-E1 cells after transfected with shRNA-NDRG2. ${ }^{* * *} P<0.001$ versus control group. ${ }^{\# \# \# ~} P<0.001$ versus shRNA-NC group.

antibodies and with second antibody for 2 hours at $25^{\circ} \mathrm{C}$. The primary antibodies included BMP2 (ab214821, Abcam, UK, dilution, 1: 1000), Runx2 (ab192256, Abcam, UK, dilution, 1: 1000), OPG (ab255723, Abcam, UK, dilution, 1: 1000), OSX (ab209484, Abcam, UK, dilution, 1: 1000), OCN (ab13402, Abcam, UK, dilution, 1: 1000), NDRG2 (ab174850, Abcam, UK, dilution, 1: 1000), p-JAK3 (\#5031, Cell Signaling Technology, Inc., USA, dilution, 1: 1000), JAK3 (ab45141, Abcam, UK, dilution, 1: 1000), p-STAT3 (ab76315, Abcam, UK, dilution, 1: 2000), STAT3 (ab119352, Abcam, UK, dilution, 1: 5000), and second antibody was anti-rabbit horseradish peroxidase-linked IgG (\#7074, Cell Signaling Technology, Inc., USA, dilution, 1: 1000). The membrane was color-developing with enhanced chemiluminescence (ECL) and detected with an ECL detection system (Thermo Fisher Scientific, Inc.). GAPDH was an internal control and the experiment was repeated three times.

\section{Cell transfection}

MC3T3-E1 cells were seeded in 6-well plates and placed in $37^{\circ} \mathrm{C}$ incubators for further culture. When the cells grew to $50 \%$ confluence, then pcDNA-NC, pcDNA-NDRG2, shRNA-NC, shRNA-NDRG2-1, and shRNA-NDRG2-2 were transfected with Lipofectamine 2000 transfection reagent, respectively.
MC3T3-E1 cells receiving no transfection were recorded as control group. These MC3T3-E1 cells were continually cultured in $37^{\circ} \mathrm{C}$ incubators.

\section{Alkaline phosphatase (ALP) activity assay}

Alkaline phosphatase (ALP) activity was measured by ALP activity kit (Beyotime, Shanghai, China). The absorbance value was determined by enzyme-linked immunoassay at the wavelength of $490 \mathrm{~nm}$ and ALP was evaluated according to the absorbance value.

\section{Alizarin red staining}

The mineralization nodules were assessed by alizarin red staining assay. After PBS washed cells with PBS for 3 times, $4 \%$ paraformaldehyde was added to fix the cells and the cells were stained at $25^{\circ} \mathrm{C}$ for 30 minutes. After PBS washed the cells twice, $2 \%$ alizarin red stain was added to stain the cells. The cells were stained at $25^{\circ} \mathrm{C}$ for 15 minutes and the staining fluid was removed. The cells were washed with distilled water for 3 times and the staining results were observed. 


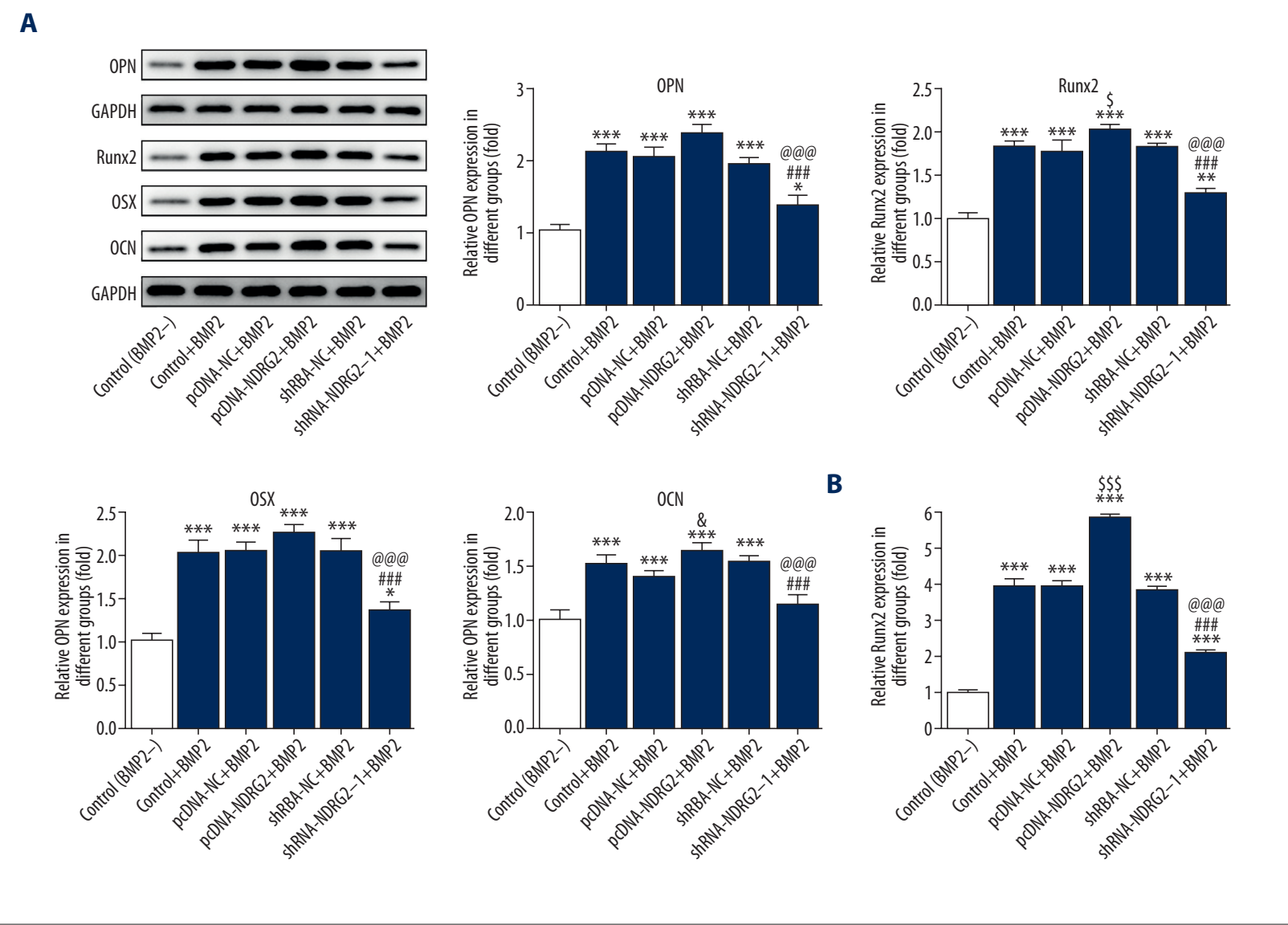

Figure 3. Upregulation and downregulation of NDRG2 affected the osteoblast differentiation. (A) NDRG2 upregulation promoted the expression of Runx2, OPG, OSX, and OCN while NDRG2 downregulation reversed. ${ }^{*} P<0.05$, ${ }^{* *} P<0.01$ and ${ }^{* * *} P<0.001$ versus control (BMP2-) group. ${ }^{\$} P<0.05$ versus pcDNA-NC+BMP2 group. ${ }^{\# \# ~} P<0.001$ versus $p c D N A-N D R G 2+B M P 2$ group. @@@ $P<0.001$ versus shRNA-NC+BMP2 group. (B) NDRG2 upregulation promoted the expression of ALP while NDRG2 downregulation reversed. * $P<0.05$ versus control (BMP2-) group. ${ }^{\$ \$} P<0.001$ versus $p C D N A-N C+B M P 2$ group. \#\#\# $P<0.001$ versus pcDNA-NDRG2+BMP2 group. ${ }^{\Delta \Delta} P<0.001$ versus shRNA-NC+BMP2 group.

\section{Statistical analysis}

SPSS 22.0 statistical software was used for data analysis. The experimental results were in the form of mean \pm standard deviation. We used the $t$-test can compare the difference between 2 groups. One-way analysis of variance (ANOVA) can analyze the difference between the multiple groups with least significant difference (LSD)-t method. $P<0.05$ meant that the difference was statistically significant.

\section{Results}

\section{NDRG2 expression is increased in BMP2-induced MC3T3-E1 cells}

The differentiation of MC3T3-E1 cells induced by BMP2 at day 0,7 , and 14 was observed by a microscope. The results of Figure $1 \mathrm{~A}$ indicated that MC3T3-E1 cells differentiation was increased with the induction time of BMP2. And the expression of cell differentiation-related proteins (Runx2, OPG, OSX, and OCN) was all increased with the induction time of BMP2 (Figure 1B-1F). With the induction time of BMP2 for MC3T3-E1 cells, NDRG2 expression was obviously increased in BMP2-induced MC3T3-E1 cells (Figure 1G, 1H).

\section{Upregulation and downregulation of NDRG2 affects the osteoblast differentiation}

Compared with the control group and pcDNA-NC group, NDRG2 expression was upregulated in MC3T3-E1 cells transfected with pcDNA-NDRG2 (Figure 2A, 2B) and decreased in MC3T3-E1 cells transfected with shRNA-NDRG2-1 and shRNA-NDRG2-2 (Figure 2C). And, NDRG2 expression in shRNA-NDRG2-1 group was less than that in shRNA-NDRG2 group (Figure 2C, 2D). Therefore, shRNA-NDRG2-1 was selected 


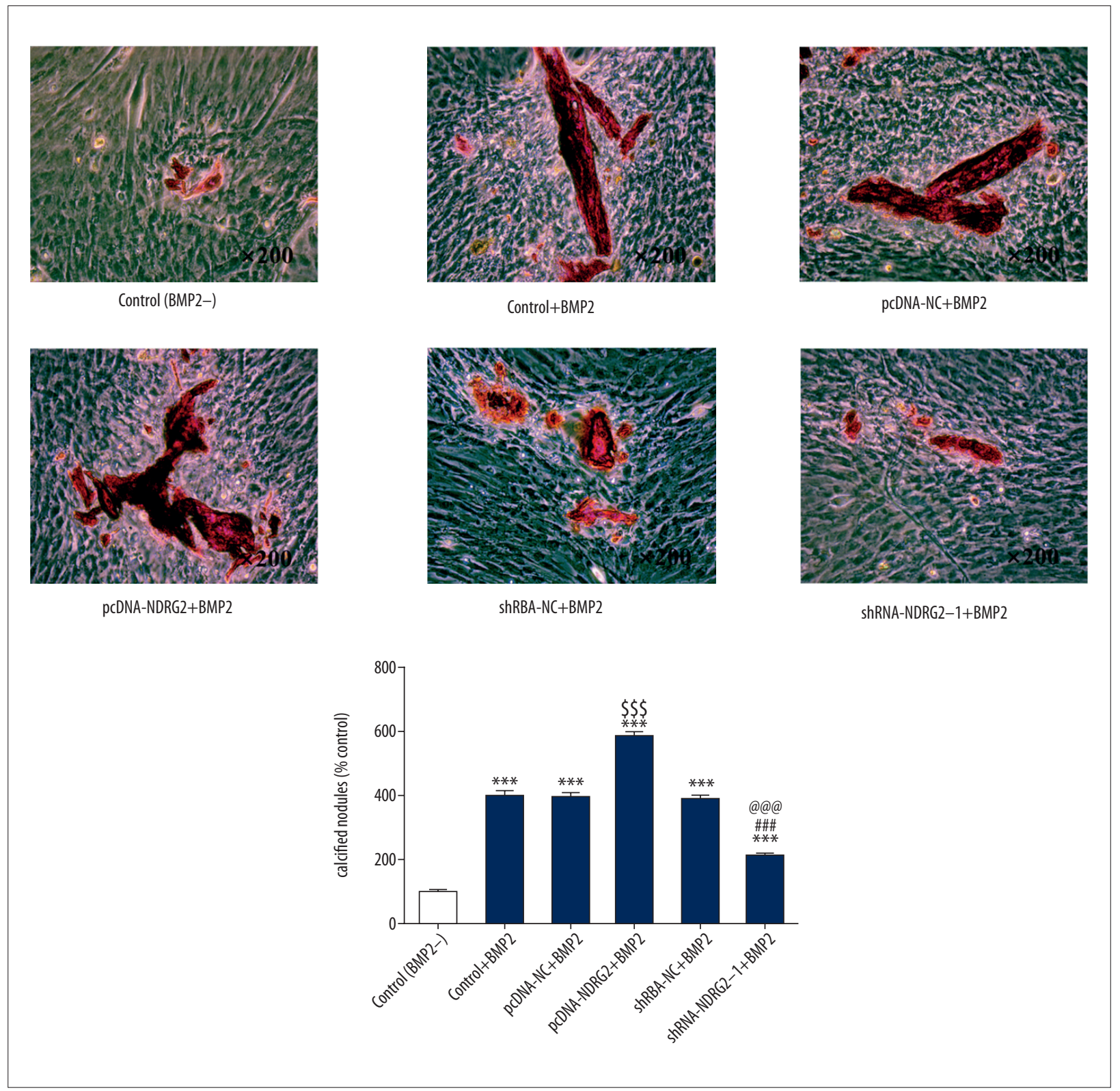

Figure 4. Upregulation and downregulation of NDRG2 affects the osteoblast calcification. NDRG2 upregulation promoted osteoblast calcification while NDRG2 downregulation reversed. ${ }^{* * *} P<0.001$ versus control (BMP2-) group. $\$ \$ \$ P<0.001$ versus pcDNA-NC+BMP2 group. \#\# $P<0.001$ versus pcDNA-NDRG2+BMP2 group. ${ }^{\circledR @} P<0.001$ versus shRNA-NC+BMP2 group.

for the subsequent experiment. As shown in Figure 3A, BMP2 induction could promote the expression of Runx2, OPG, OSX, and OCN in BMP2-induced MC3T3-E1 cells. And NDRG2 upregulation further increased the expression of Runx2, OPG, OSX, and OCN in BMP2-induced MC3T3-E1 cells. However, NDRG2 downregulation decreased the expression of Runx2, OPG, OSX, and OCN in BMP2-induced MC3T3-E1 cells. As shown in Figure 3B, the ALP expression was increased when the MC3T3-E1 cells were treated with BMP2 and NDRG2 upregulation further promoted the ALP expression in BMP2-induced MC3T3-E1 cells. However, NDRG2 downregulation inhibited the ALP expression in BMP2-induced MC3T3-E1 cells. Therefore, NDRG2 overexpression could promote the osteoblast differentiation while NDRG2 downregulation was the opposite.

\section{Upregulation and downregulation of NDRG2 affects the osteoblast calcification}

BMP2 could increase the calcium deposit in MC3T3-E1 cells and NDRG2 upregulation further promoted the calcium deposit in BMP2-induced MC3T3-E1 cells. However, NDRG2 downregulation decreased the calcium deposit in BMP2-induced MC3T3-E1 


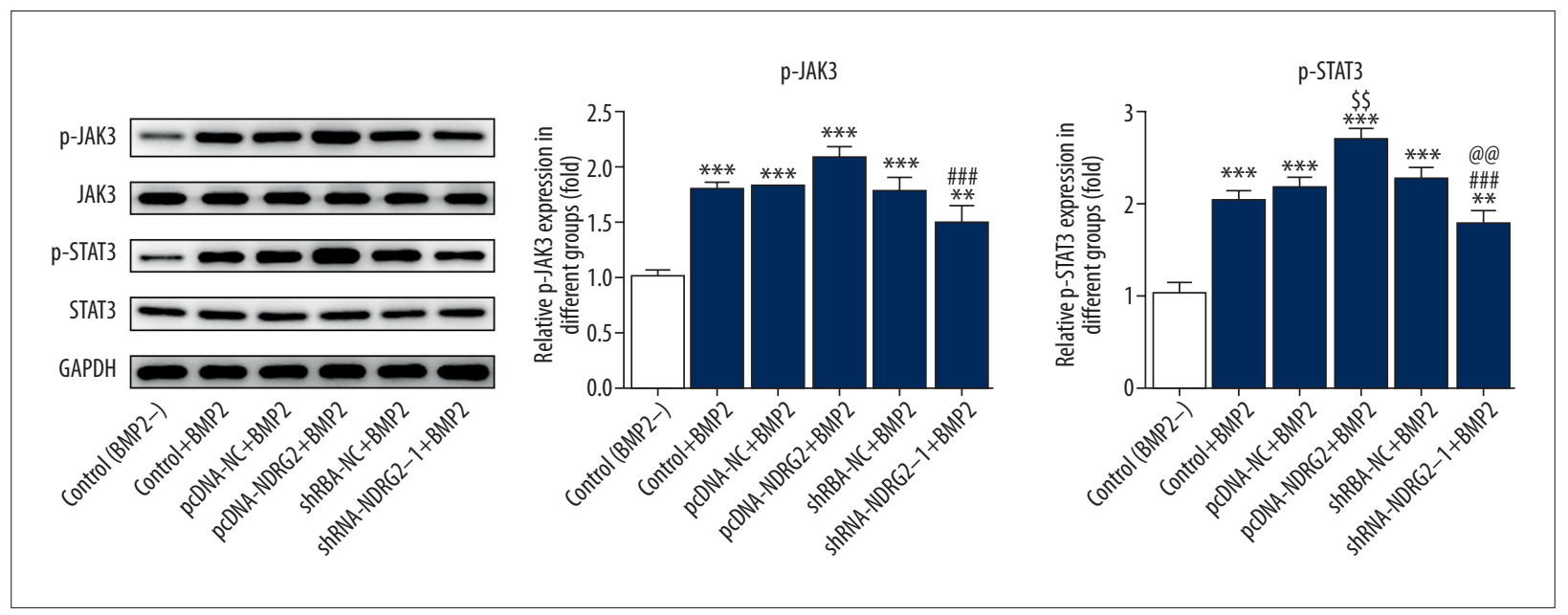

Figure 5. Upregulation and downregulation of NDRG2 affects the JAK3/STAT3 signaling pathway. NDRG2 upregulation promoted the expression of $p$-JAK3 and $p$ - STAT3 while NDRG2 downregulation reversed. ${ }^{* *} P<0.01$ and ${ }^{* * *} P<0.001$ versus control (BMP2-) group. $\$ \$ P<0.01$ versus pcDNA-NC+BMP2 group. ${ }^{\# \#} P<0.001$ versus pcDNA-NDRG2+BMP2 group. ${ }^{\circledR} P<0.01$ versus shRNA-NC+BMP2 group.

cells (Figure 4). Therefore, NDRG2 upregulation could increase the osteoblast calcification while NDRG2 downregulation was the opposite.

\section{Upregulation and downregulation of NDRG2 affects the JAK3/STAT3 signaling pathway}

As shown in Figure 5, the phosphorylation levels of JAK3 and STAT3 were increased in BMP2-induced MC3T3-E1 cells. In addition, NDRG2 upregulation promoted the phosphorylation levels of JAK3 and STAT3 in BMP2-induced MC3T3-E1 cells. However, NDRG2 downregulation inhibited the phosphorylation levels of JAK3 and STAT3 in BMP2-induced MC3T3-E1 cells. Therefore, NDRG2 upregulation could activate the JAK3/STAT3 signaling pathway while NDRG2 downregulation was the opposite.

\section{NDRG2 promotes BMP2-induced osteoblast differentiation through the JAK3/STAT3 signaling pathway}

WHI-P154 is a signaling inhibitor of JAK3/STAT3 signaling pathway. The BMP2-induced MC3T3-E1 cells transfected with pcDNA-NDRG2 were respectively treated with $20 \mu \mathrm{M}$ and $50 \mu \mathrm{M}$ WHI-P154 for day 14. WHI-P154 could suppressed the expression of BMP2, Runx2, OPG, OSX, and OCN. The inhibition effects of WHI-P154 were gradually enhance with the increase of WHI-P154 concentration for MC3T3-E1 cells (Figure 6A). The ALP expression was decreased when MC3T3-E1 cells treated with WHI-P154 and the expression of ALP in pcDNA-NDRG2+BMP2+WHI-P154 $(50 \mu \mathrm{M})$ group was less than that in PcDNA-NDRG2+BMP2+WHI-P154 (20 $\mu \mathrm{M})$ group (Figure $6 \mathrm{~B}$ ). As shown in Figure $6 \mathrm{C}$, the intracellular calcium deposit was decreased when MC3T3-E1 cells treated with WHI-P154 and the intracellular calcium deposit in
pcDNA-NDRG2+BMP2+WHI-P154 $(50 \mu \mathrm{M})$ group was less than that in pcDNA-NDRG2+BMP2+WHI-P154 $(20 \mu \mathrm{M})$ group. The inhibition of JAK3/STAT3 signaling pathway could decrease the expression of BMP2, Runx2, OPG, OSX, OCN, and ALP and calcium deposit. Therefore, NDRG2 accelerated BMP2-induced osteoblast differentiation by the JAK3/STAT3 signaling pathway.

\section{Discussion}

The effect of NDRG2 on osteoblastic differentiation and calcification through the JAK3/STAT3 signaling pathway was explored. The experimental results indicated that NDRG2 promoted BMP2-induced osteoblastic differentiation and calcification by activating JAK3/STAT3 signaling pathway.

According to the International Osteoporosis Foundation (IOF), about $15 \%$ to $30 \%$ of men was affected by osteoporosis while the ratio in women could reach to $30 \%$ to $50 \%$, who needed to pay special attention to osteoporosis prevention and treatment at the right age [10]. Hormone replacement treatment (HRT) is usually used in postmenopausal osteoporosis (PMOP) therapy. However, long-term use of chemical synthetic hormones may cause hyperplasia and cancerization of the reproductive system and the occurrence of cardiovascular and cerebrovascular diseases [11-13].

BMP2 is the only growth factor inducing the mesenchymal cells to differentiate into bone tissues. In the early stage of bone formation, BMP2 can not only recruit undifferentiated mesenchymal cells into bone formation to differentiate into bone cells, but also reverse the differentiation of fibroblast myoblasts and bone marrow basal cells into bone cells [14]. 


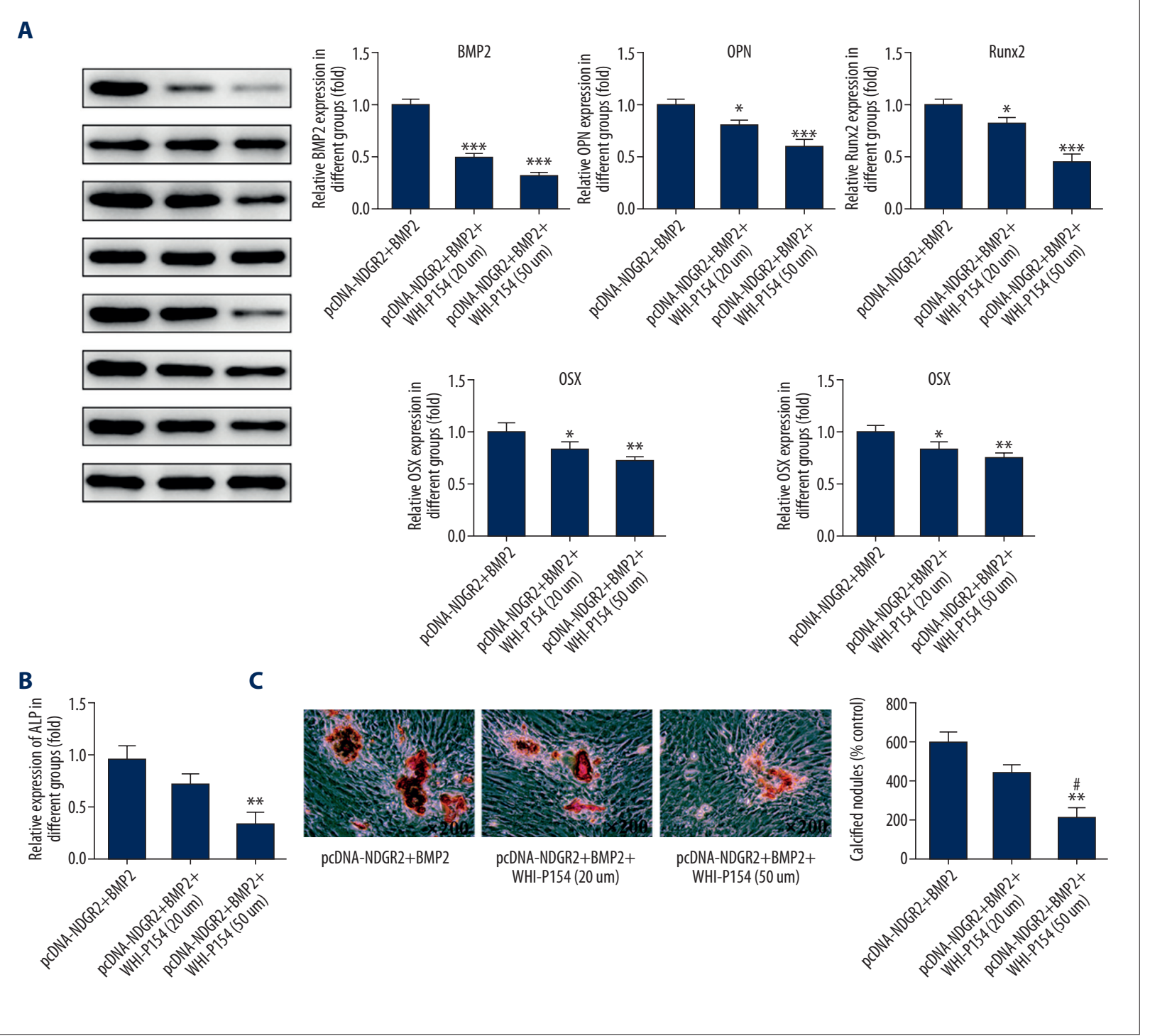

Figure 6. NDRG2 promoted BMP2-induced osteoblast differentiation through the JAK3/STAT3 signaling pathway. (A) The expression of BMP2, Runx2, OPG, OSX, and OCN was decreased in MC3T3-E1 cells after treated with WHI-P154. ${ }^{*} P<0.05,{ }^{* *} P<0.01$ and ${ }^{* * *} P<0.001$ versus pCDNA-NDRG2+BMP2 group. (B) The expression of ALP was decreased in MC3T3-E1 cells after treated with WHI-P154. ${ }^{* *} P<0.01$ versus pcDNA-NDRG2+BMP2 group. (C) The osteoblast calcification was decreased in MC3T3-E1 cells after treated with WHI-P154. ${ }^{* *} P<0.01$ versus pCDNA-NDRG2+BMP2 group. ${ }^{*} P<0.05$ versus pcDNA-NDRG2+BMP2+WHI-P154 (50 um).

In this study, the differentiation of MC3T3-E1 cells treated with BMP2 were increased. Another study found that during embryonic development, NDRG2 was expressed in the heart, brain, intestine, liver, kidney, skeletal muscle, cartilage, and chorion [15]. With the development of research, existing literature reported that NDRG2 could regulate the formation and development of vertebrae in the embryonic stage, which suggested that NDRG2 was closely related to the occurrence and development of bone. NDRG2 gene is mainly expressed in mesenchymal stem cells (MSC) which are related to the early stage of limb buds. After mesenchymal stem cells begin to differentiate into cartilage and bone, NDRG2 is mainly expressed in perichondrium [16]. In the present study, NDRG2 overexpression promoted the osteoblast differentiation and calcification while NDRG2 downregulation was the opposite, which was consistent with the previous study.

Activation of JAK2/STAT3 signal channels can regulate the expression of angiogenesis factors [17-19]. Study demonstrated that AG490, as a JAK2/STAT3 specificity inhibitor, could inhibit the expression of JAK2/STAT3. The level of JAK2/STAT3 decreased, leading to the decrease of BMP2 protein content in 
microenvironment of bone tissue [20]. Here, we demonstrated that NDRG2 upregulation could activate the JAK3/STAT3 signaling pathway while NDRG2 downregulation was the opposite. Therefore, NDRG2 promoted BMP2-induced osteoblast differentiation through the JAK3/STAT3 signaling pathway.

\section{Conclusions}

NDRG2 expression was increased in osteogenic differentiation. NDRG2 upregulation could promote the osteoblast differentiation and calcification by activating JAK3/STAT3 signaling pathway. These results suggested that NDRG2 may be as a potential drug to prevent and treat the osteoporosis. However, only MC3T3-E1 cells were examined in current study; therefore, similar results in other cells would be required to explore how NDRG2 regulates the osteoblast differentiation and calcification.

\section{Conflicts of interest}

None.

11. Tit DM, Bungau S, lovan C et al: Effects of the hormone replacement therapy and of soy isoflavones on bone resorption in postmenopause. J Clin Med, 2018; 7(10): pii: E297

12. Zuo HL, Sun AJ, Gao LH et al: Effect of menopausal hormone therapy on bone mineral density in Chinese women: A 2-year, prospective, open-label, randomized-controlled trial. Med Sci Monit, 2019; 25: 819-26

13. Lou S, Lv H, Yin P et al: Combination therapy with parathyroid hormone analogs and antiresorptive agents for osteoporosis: A systematic review and meta-analysis of randomized controlled trials. Osteoporos Int, 2019 30(1): 59-70

14. Kim K-M, Kim D-Y, Lee D-S et al: Peroxiredoxin II negatively regulates BMP2-induced osteoblast differentiation and bone formation via PP2A C $\alpha$ mediated Smad1/5/9 dephosphorylation. Exp Mol Med, 2019; 51(6): 62

15. Hu XL, Liu XP, Deng YC et al: Expression analysis of the NDRG2 gene in mouse embryonic and adult tissues. Cell Tissue Res, 2006; 325(1): 67-76

16. Zhu H, Zhao J, Zhou W et al: Ndrg2 regulates vertebral specification in differentiating somites. Dev Biol, 2012; 369: 308-18

17. Johannson HR, Zywiel MG, Marker DR et al: Osteonecrosis is not a predic tor of poor outcomes in primary total hip arthroplasty: A systematic literature review. Int Orthop, 2011; 35(4): 465-73

18. Kang DY, Sp N, Kim DH et al: Salidroside inhibits migration, invasion and angiogenesis of MDA-MB 231 TNBC cells by regulating EGFR/Jak2/STAT3 signaling via MMP2. Int J Oncol, 2018; 53(2): 877-85

19. Liu JF, Deng WW, Chen L et al: Inhibition of JAK2/STAT3 reduces tumor-induced angiogenesis and myeloid-derived suppressor cells in head and neck cancer. Mol Carcinog, 2018; 57(3): 429-39

20. Weng $Y$, Jiang W, Zhou Y: [The effect of JAK2/STAT3 signaling pathway on cytokines in synovial tissues of steroid-induced femur head necrosis rats.] Fujian Medical Journal, 2019; 41(02): 131-34 [in Chinese] 